\title{
Abdominal Aortic Aneurysm (AAA) in the elderly: endovascular versus open surgical repair
}

\author{
R Russo \\ From de Senectute: Age and Health Forum \\ Catanzaro, Italy. 5-7 December 2009
}

\section{Background}

The traditional surgical intervention of open aneurysmectomy and prosthetic graft is still now the gold standard for the treatment of abdominal aortic aneurysm. Today, the new mini-invasive endovascular approach is a valid therapeutic option to customize the treatment based on clinical characteristics of patient and morphological features of the aneurysmal lesion. The purpose of this study is to establish which technique is more suitable for the treatment of the AAA in the elderly patients.

\section{Materials and methods}

100 patients aged between 65 and 75 (M:F 85:15) were enrolled in 3 years. After a preoperative cardiac screening, patients were divided into two groups of 50 patients. In group I were included high risk patients (acute renal insufficiency, chronic obstructive pulmonary disease, hypertension and valvular defects), while in group II were included low risk patients with not relevant pathologies. All patients of group I underwent endovascular and surgical treatment, patients in group II were submitted to "open" surgical intervention because of moderate risk.

\section{Results}

Of the 50 patients of group I, 2 deaths have occurred in the immediate post-operative period for pre-existing comorbidities. The follow-up at 12 months with eco Doppler ultrasonography showed the absence of endoleak or an increase in the volume of AAA in 45 patients, while for the last 3 patients an open laparotomy was performed for a conversion with a prosthetic graft immediately after the endovascular procedure. In the immediate post-operative period, we registered 4 deaths among patients of group II due to cardiac arrhythmic or

Chair of General Surgery, Vascular Surgery Training Programme, University Magna Graecia of Catanzaro, Italy
Table 1

\begin{tabular}{lll}
\hline & Traditional Surgery & Endovascular Approach \\
\hline Age & $<70$ & $>70$ \\
Sex & M $>$ F & M $>\mathrm{F}$ \\
Disorders preoperative & Absent & Present \\
Follow up 7 days & 4 & 2 \\
Follow up 12 months & 3 paresis of ileum & 3 reinterventions \\
\hline
\end{tabular}

ischemic complications or lung failure. The follow up at 12 months has shown paresis of the ileum in 3 patients; the other 43 had a normal post-operative course, Table 1 .

\section{Conclusions}

The study shows that the minimally invasive and endovascular method represents a valid therapeutic option especially in high risk patients with a reduction in immediate post-operative complications. The one year follow-up has revealed the normal predictable incidence of complications.

Published: 19 May 2010

Reference

1. Lederle FA, Freischlag JA, Kyriakides TC, Padberg FT Jr, Matsumura JS, Kohler TR, Lin PH, Jean-Claude JM, Cikrit DF, Swanson KM, Peduzzi PN: Outcomes following endovascular vs open repair of abdominal aortic aneurysm: a randomized trial. Open Versus Endovascular Repair (OVER) Veterans Affairs Cooperative Study Group. 2009, 302(14):1535-42.

doi:10.1186/1471-2318-10-S1-A75

Cite this article as: Russo: Abdominal Aortic Aneurysm (AAA) in the elderly: endovascular versus open surgical repair. BMC Geriatrics 2010 10(Suppl 1):A75. 